\title{
Contact screening in tuberculosis: can we identify those with higher risk?
}

\section{To the Editor:}

Contact screening, as a strategy to identify recently infected individuals, is part of the tuberculosis (TB) elimination strategy. It follows risk stratification concerning the infectiousness of the index patient, the duration and proximity of exposure, and the susceptibility of the contact [1, 2]. For its optimisation it is important to know which risk factors are associated with Mycobacterium tuberculosis transmission in order to not over screen or lose at-risk contacts. In view of that, this study aims to identify potential risk factors for $M$. tuberculosis transmission among contacts of pulmonary $\mathrm{TB}$ patients, in a Portuguese TB reference centre.

From January to December of 2011, all contacts of confirmed pulmonary TB patients, screened in the TB centre, were questioned about their exposure to the index patient through a questionnaire completed during the medical appointment. Both household and casual contacts were screened, independent of their cumulative exposure.

Contacts excluded: those with exposure outdoors (e.g. in the street); those with incomplete characterisation of exposure (e.g. index patients for whom we did not know the symptomatic period); those non-compliant with screening (e.g. contacts who failed tuberculin skin test (TST) reading); and those with a past history of M. tuberculosis infection or TB disease (e.g. contacts verbal report of previous TB disease, contact with a pulmonary TB patient or treatment for latent tuberculosis infection (LTBI)).

Contacts of patients with a diagnosis of pulmonary TB were identified for screening at the moment of the index patient diagnosis. The index patient was asked to identify contacts within the family, social and work spheres. Furthermore, some other contacts were identified by the public health unit during a visit to home, work and leisure places. Contacts were then asked to call for an appointment in the TB centre. Contact screening included evaluation for possible TB disease with a symptoms questionnaire (e.g. cough, chest pain, fever, night sweats, appetite loss or weight loss) and chest radiography. After exclusion of TB disease, TST was performed and if the result was positive an interferon $\gamma$ release assay (IGRA) was carried out. When the initial screening was negative, a re-evaluation was scheduled after 8-10 weeks. Appropriate treatment and follow-up care were proposed for those with TB disease or LTBI.

The questionnaire had three sections. Section I identified index patient characteristics and was filled with the information collected from the clinical record. Section II assessed information about the contact and the level of exposure. Section III concerned screening results. All questionnaires were performed by trained doctors in a private setting at the TB centre and contacts were guaranteed confidentiality.

Definitions used for the questionnaire are as follows.

Household contacts: those who lived in the same house as the index patient.
Casual contacts: those who did not live in the same house as the index patient; these were mostly family, workplace and school contacts.

Positive screening: TB disease or LTBI. TB disease referred to clinical and/or radiological disease confirmed with bacteriological or histological evidence. LTBI was defined as presumptive $M$. tuberculosis infection, as evidenced by a positive TST with a positive IGRA, without any sign of clinical or radiological manifest disease.

Symptomatic period: the time from onset of the first symptom i.e. cough, fever or night sweats until the diagnosis.

Size of the exposure site: "small" for an area $<20 \mathrm{~m}^{2}$ (e.g. car, bedroom, living room or office) and "large" for an area $\geqslant 20 \mathrm{~m}^{2}$ (e.g. restaurant); registered the smallest place remembered by the contact.

Exposure sites: with ventilation were those with windows and without ventilation were those without windows; registered the lowest ventilation remembered by the contact.

Univariate and multivariate logistic regression models were used to investigate the association between the occurrence of $M$. tuberculosis transmission and risk factors, measured through odds ratios and correspondent 95\% confidence intervals. Statistical significance was defined at the 0.05 level.

The study protocol was reviewed and approved by the ethics committee of the Hospital Centre of Vila Nova de Gaia/Espinho, Portugal, and all contacts provided oral informed consent.

Over the studied period, we identified 69 index patients with pulmonary TB and 741 contacts. Of these, 397 (53.6\%) were included in the analysis following inclusion criteria. There was no refusal to participate.

Positive screening was identified in $75(18.9 \%)$ contacts. The prevalence of TB disease was $0.8 \%$ (three contacts) and LTBI was $18.1 \%$ (72 contacts). Of the contacts studied, $56.7 \%$ were males with a median age of 32 years.

The multivariate analysis (table 1 ) revealed that older contact age (OR 1.03, 95\% CI 1.02-1.05), cohabitation (OR 4.26, 95\% CI $2.10-8.65$ ), positive sputum analysis (smear or culture) (OR $2.01,95 \%$ CI 1.06-3.82) and duration of the index patient symptoms (OR 1.006, 95\% CI 1.001-1.011) were independent risk factors for positive screening. No significant association was found concerning the presence of cavitations on chest radiography of the index patient, contact duration and size or ventilation of the exposure site.

In this study, $18.9 \%$ of the contacts had a positive screening. We identified older contact age, cohabitation, positive sputum analysis and duration of the index patient symptoms as independent risk factors for a positive screening. The risk of having a positive screening increased significantly for each additional 


\begin{tabular}{|c|c|c|c|}
\hline \multirow[t]{2}{*}{ TABLE 1} & \multicolumn{3}{|c|}{$\begin{array}{l}\text { Association between index patients or contacts/ } \\
\text { exposure characteristics and positive screening } \\
\text { in contacts }\end{array}$} \\
\hline & & $\begin{array}{l}\text { Crude univariate } \\
\text { OR }(95 \% \mathrm{Cl})\end{array}$ & $\begin{array}{l}\text { Adjusted multivariate } \\
\text { OR }(95 \% \mathrm{Cl})^{\#}\end{array}$ \\
\hline \multicolumn{4}{|c|}{ Index patient characteristics } \\
\hline \multicolumn{4}{|c|}{ Biological product studied } \\
\hline Bronchor & veolar lavage & 1 & 1 \\
\hline Sputum & & $1.18(0.69-2.02)$ NS & $2.01(1.06-3.82)^{\star}$ \\
\hline Symptomat & period days & $1.004(0.999-1.008)$ NS & $1.006(1.001-1.011)^{\star}$ \\
\hline \multicolumn{4}{|c|}{ Chest radiography } \\
\hline Without c & ivitation & 1 & \\
\hline Cavitatior & & $1.13(0.68-1.87)$ NS & \\
\hline \multicolumn{4}{|c|}{ characteristics } \\
\hline \multicolumn{4}{|c|}{ Sex } \\
\hline Female & & 1 & 1 \\
\hline Male & & $1.54(0.93-2.55) \mathrm{NS}$ & $1.50(0.88-2.56) \mathrm{NS}$ \\
\hline Age years & & $1.02(1.01-1.03)^{\star}$ & $1.03(1.02-1.05)^{\star \star \star}$ \\
\hline \multicolumn{4}{|c|}{ Comorbidities } \\
\hline \multicolumn{4}{|c|}{ Diabetes mellitus } \\
\hline No & & 1 & \\
\hline Yes & & $2.60(1.15-5.89)^{*}$ & \\
\hline \multicolumn{4}{|c|}{ Chronic renal failure } \\
\hline No & & 1 & \\
\hline Yes & & $0.91(0.42-1.96)$ NS & \\
\hline \multicolumn{4}{|c|}{ Contact type } \\
\hline Casual & & 1 & 1 \\
\hline Househo & & $4.86(2.46-9.63)^{\star \star \star}$ & $4.26(2.10-8.65)^{\star \star \star}$ \\
\hline Exposure d & ration $\mathrm{h}$ & $1.001(1.000-1.001)$ NS & \\
\hline \multicolumn{4}{|c|}{ Sleeping together } \\
\hline No & & 1 & \\
\hline Yes & & $5.35(1.99-14.39)^{*}$ & \\
\hline \multicolumn{4}{|c|}{ Eating together } \\
\hline No & & 1 & \\
\hline Yes & & $1.62(0.97-2.69) \mathrm{NS}$ & \\
\hline \multicolumn{4}{|c|}{ Size of the exposure site } \\
\hline "Large" & & 1 & \\
\hline "Small" & & $1.03(0.55-1.93)$ NS & \\
\hline \multicolumn{4}{|c|}{ Ventilation of the exposure site } \\
\hline Yes & & 1 & \\
\hline No & & $1.13(0.47-2.69)$ NS & \\
\hline
\end{tabular}

\#: the final binomial logistic model had biological product studied, symptomatic period, sex, age and contact type as explanatory variables. ${ }^{*}: \mathrm{p}<0.05 .{ }^{* * *}$ : $p<0.001$. Ns: nonsignificant at the 0.05 level.

year of age of the contact $(3.0 \%)$ and for each additional day in the duration of the index patient symptoms $(0.6 \%)$.

A similar rate of TB disease $(0.8 \%$ versus $0.6 \%$ ) and LTBI $(18.1 \%$ versus $17.2 \%$ ) was observed in a recent report in Mississippi, USA [3].

MADEBO et al. [4] observed that patients become more contagious as the delay in diagnosis and treatment of TB progresses. We estimated an increase in the odds of positive screening among contacts of $0.6 \%$ for every day that the diagnosis of the index patient is delayed.

Increased risk of $M$. tuberculosis transmission was shown for the presence of positive sputum smear or culture. Previous studies have also noted that infectiousness is highest when the sputum is positive [2,5-7]. In our study, however, cavitation on chest radiography of the index patient was not a predictor of positive screening. This result is not accordant with others $[2,6,8]$. Cavitations may be related to the symptomatic period before diagnosis.

Increased risk of M. tuberculosis transmission was shown for households, which is already well known [2].

Contacts of older age were more likely to have a positive screening. In order to exclude past infection, we excluded all contacts with a previous history of TB or past exposure to TB disease. Other studies, namely from low TB incidence countries, have also shown a greater risk for positive screening among the oldest [9, 10] strengthening the hypothesis of greater host susceptibility.

In our study, we also found that exposure duration, size and ventilation of the exposure site had no effect on the risk of $M$. tuberculosis transmission, while previous studies stated the opposite [1, 2]. Although it is very difficult to analyse this exposure due to recall bias, we asked the contacts to remember the smallest and least ventilated place they shared with the index patient. In that sense, it would be over-estimated and not under-estimated.

All comorbidities, including HIV, immunosuppressive treatment, alcoholism and illegal drug use were self-reported by contacts. Information bias in ascertainment of these variables cannot be excluded in our study; however, if a nondifferential bias had occurred, the true association would even be higher than the reported one.

The main strengths of this research were the high participation rate, the large number of contacts analysed, and the inclusion of contacts from all socioeconomic strata and the broad range of contacts-age.

Additional efforts should be made to ensure that contacts with these risk factors have priority in screening.

\section{Maria Aurora Mendes*, Rita Gaio", ${ }^{\#,}$ Ricardo Reis ${ }^{+}$and Raquel Duarte ${ }^{\S, f, * *, \# \#}$}

*Medical School, University of Porto, "Dept of Mathematics, Sciences School, University of Porto, "Centre of Mathematics, University of Porto, ${ }^{* *}$ Institute of Public Health, University of Porto, and \#\# Dept of Clinical Epidemiology, Predictive Medicine and Public Health, Medical School, University of Porto, Porto, ${ }^{+}$Hospital Centre of Trás-os-Montes and Alto Douro, Vila Real, ${ }^{\S}$ Pulmonology Diagnosis Centre, Vila Nova de Gaia, and ${ }^{f}$ Hospital Centre of Vila Nova de Gaia/Espinho, Vila Nova de Gaia, Portugal.

Correspondence: M. A. Mendes, Medical School, University of Porto, Alameda Prof Hernâni Monteiro, 4200-319 Porto, Portugal. E-mail: mimed07090@med.up.pt

Statement of Interest: None declared. 
Acknowledgements: The authors wish to thank the staff of the Portuguese TB reference centre (Pulmonology Diagnosis Centre, Vila Nova de Gaia) for making this study possible.

\section{REFERENCES}

1 Erkens CGM, Kamphorst M, Abubakar I, et al. Tuberculosis contact investigation in low prevalence countries: a European consensus. Eur Respir J 2010; 36: 925-949.

2 Guidelines for the Investigation of Contacts of Persons with Infectious tuberculosis. Recommendations from the National Tuberculosis Controllers Association and CDC. MMWR Recomm Rep 2005; 54: 1-47.

3 Webb RM, Holcombe M, Pearson MM. Tuberculosis contact investigation in a rural state. Int J Tuberc Lung Dis 2003; 7: S353S357.

4 Madebo T, Lindtjorn B. Delay in treatment of pulmonary tuberculosis: an analysis of symptom duration among Ethiopian patients. MedGenMed 1999; E6.
5 Nava-Aguilera E, Andersson N, Harris E, et al. Risk factors associated with recent transmission of tuberculosis: systematic review and meta-analysis. Int J Tuber Lung Dis 2009; 13: 17-26.

6 Reichler MR, Reves R, Bur S, et al. Evaluation of investigations conducted to detect and prevent transmission of tuberculosis. JAMA 2002; 287: 991-995.

7 Verhagen LM, Van Den Hof S, Van Deutekom H, et al. Mycobacterial factors relevant for transmission of tuberculosis. J Infect Dis 2011; 203: 1249-1255.

8 Marks SM, Taylor Z, Qualls NL, et al. Outcomes of contact investigations of infectious tuberculosis patients. Am J Respir Crit Care Med 2000; 162: 2033-2038.

9 Mandal P, Craxton R, Chalmers JD, et al. Contact tracing in pulmonary and non-pulmonary tuberculosis. QJM 2012; 105: 741-747.

10 Tuberculosis Research Centre Indian Council of Medical Research. Risk of tuberculosis among contacts of isoniazid-resistant and isoniazid-susceptible cases. Int J Tuberc Lung Dis. 2011; 15: 782-788.

DOI: $10.1183 / 09031936.00164612$

\section{Evidence of respiratory system remodelling in a competitive freediver}

\section{To the Editor:}

We present a healthy freediving subject with increasing lung volumes associated with repeated use of a technique used to enhance athletic performance. The repeated use of the technique over time appears to have altered respiratory system mechanics without any functionally important macroscopic lung damage, at least as evidenced by computed tomography scans and measures of gas exchange.

Glossopharyngeal insufflation (GI) employs the glossopharyngeal structures to force air into the lungs above total lung capacity (TLC). It was developed to assist patients with diaphragm weakness in the era when polio was common [1]. Competitive freedivers have modified this technique to increase lung gas prior to apnoea [2], which has proven to enhance duration, distance and depth achieved while submersed.

Lung barotrauma has been associated with GI [3,4], which raises the possibility that use of this technique results in significant lung damage and long-term physiological impairment.

The research data from a healthy competitive freediver who practised regular GI training was reviewed. This included longitudinal respiratory function and computed tomography images.

On initial presentation in 2004, the subject was a 25-year-old male $(186 \mathrm{~cm}, 90 \mathrm{~kg})$, healthy with no known respiratory or cardiac disease and was not taking any medications. A recreational spearfisher since 1999, he had taken up freediving and regular GI training in 2002. Training sessions increased to three times weekly by 2009, a level that was maintained through to 2012 and he consistently competed at a national level. The subject's static apnoea performance (breath-hold time) had increased from $7 \mathrm{~min}$ in 2004 to $8 \mathrm{~min}$ in 2012 and there has been a $37 \mathrm{~m}$ increase in ocean depth achieved (unassisted with fins) to $88 \mathrm{~m}$. He had been in good health in this period without proven barotrauma.

Complex respiratory function tests prior to, and immediately following, maximal GI were reviewed. Measurements were taken on four occasions over a period of 8 years (from age 25 to 33 years). All tests were performed according to American Thoracic Society/European Respiratory Society criteria [5-7] and were conducted by the same scientific officer with one equipment upgrade (Vmax Encore, Sensormedics, Yorba Linda, CA, USA). Lung volumes immediately following maximal GI (TLC $\mathrm{GI}$ ) were measured using a technique previously described [2]. Measurements were made using a plethysmograph, recording exhaled gas volumes rather than Euclidian (geometric) volumes, thus being free of a gas compression effect. Reference values were derived from European Community for Coal and Steel [8].

Previous studies of freedivers, spearfishers and swimmers have noted overall large lung volumes when compared to normal. This subject was no exception. In addition, he is adept at GI and was able to entrain an additional $2.4 \mathrm{~L}$ of gas achieving a vital capacity (VC) following GI equivalent to $192 \%$ of predicted in 2004. By 2012 the additional lung gas volume from GI had reduced to $1.62 \mathrm{~L}$, in proportion to the increase in VC.

Over the review period, an increase in measured lung volumes is evident with a total $>800 \mathrm{~mL}$ increase in $\mathrm{VC}$, functional residual volume (FRC) and TLC (table 1). There is no evidence 